\title{
The Role of Multidetector CT in Revised Atlanta Classification of Acute Pancreatitis
}

\author{
Hesham M. Farouk, Eman F. Abdelkhalik, Nesma S. Ellithy
}

\begin{abstract}
Department of Radiology, Benha faculty of medicine, Banha University, Egypt.
\end{abstract}

Correspondence to: Nesma S. Ellithy, Department of Radiology, Benha faculty of medicine, Banha University, Egypt.

Email:

dr.nesmasaied@gmail.com

Received: 22 August 2021

Accepted: 30 November 2021

\begin{abstract}
:
Background: CT has become the standard of choice and worldwide the most commonly used imaging modality for the initial evaluation of acute pancreatitis and its sequelae. Aim: The aim of this study is to asses the role of multidetector CT in diagnosis of acute pancreatitis according to Revised Atlanta classification of acute pancreatitis. Patients and Methods: This study included 30 patients with previously diagnosed as acute pancreatitis attacks, clinically suspected acute pancreatitis, laboratory results suggesting acute pancreatitis to do multidetector CT. Results: Our study confirms that contrast enhanced $\mathrm{CT}$ is a reliable and accurate technique to determine type of acute pancreatitis according to The Revised Atlanta classification. Conclusion: Our data and in agreement with the previous studies has demonstrated that contrast enhanced CT is a reliable and accurate technique to determine type of acute pancreatitis according to The Revised Atlanta classification. Treatment planning
\end{abstract} is based on severity of pancreatitis and presence or absence of infection combined with clinical signs. The revised Atlanta classification system with CT helps guide management and monitors the success of treatment.

Key words: CT, Revised Atlanta Classification, Acute pancreatitis

\section{Introduction:}

Acute pancreatitis is an acute inflammatory condition, with a range of severity as well as various local and systemic complications. Gallstones and alcohol are the first and second most common causes of acute pancreatitis [1]. According to the Atlanta Classification, 2 out of 3 features are required for diagnosing acute pancreatitis: (1) acute-onset upper abdominal pain radiating to the back; (2) serum lipase or 
amylase levels 3 or more times higher than the normal range; and (3) characteristic findings of acute pancreatitis on contrastenhanced computed tomography (CT) and less commonly magnetic resonance imaging (MRI) or ultrasound [2]. In the 1992 Atlanta classification was established which identified two categories of AP, "mild" and "severe" and recommended the clinical treatment for each type.

However, a subgroup of AP patients who fell in-between the two 1992 severity categories were often observed to have relatively good outcomes and respond positively to less aggressive treatment protocols than those with severe disease, thus calling for the re vision of the existing classification system. In 2012 the Atlanta classification was revised by adding a third category defined as " moderately severe [3] .

The revised Atlanta classification (RAC) was issued in 2012. It introduces new terminology to designate AP types and stages, collections that develop during AP, and degrees of severity [4] .

The incidence of acute pancreatitis continues to increase worldwide, in parallel with an increasing demand on imaging resources to evaluate the severity of disease.
Imaging modalities available for assessment of acute pancreatitis include conventional radiography, abdominal ultrasound (US), multidetector computed tomography (CT), and magnetic resonance imaging (MRI) [5] . Of these, CT has become the standard of choice and worldwide the most commonly used imaging modality for the initial evaluation of acute pancreatitis and its sequelae [6].

The RAC differentiates interstitial edematous pancreatitis (IEP) and necrotizing pancreatitis, which in turn is classified as parenchymal necrosis, peripancreatic necrosis, or both. Pancreatic and peripancreatic collections are named based on two criteria, namely, whether they develop within or after the first 4 weeks and whether they contain fluid only or also solid material [7].

\section{Patients and Methods:}

This case control study included 30 patients; 16 males and 14 females. Their age ranged between 21 to 70 years.

The included patients were referred from Shebin-Elkom National liver institute to do CT for better evaluation, during the period from April 2019 to April 2021 with acute abdominal pain mainly epigastric radiating to back, .Patient presenting with elevated serum amylase and lipase level. 
Patients with contraindication to CT Pregnant and lactating patient, renal impairment (serum creatinine level above the reference $1.5 \mathrm{mg} / \mathrm{dL}$ ), cardiac failure and previous allergic reaction to contrast media.

All the patients were subjected to full history taking, clinical examination, laboratory investigations (CBC, Serum amylase, Serum lipase ,Serum triglycerides ,Renal function tests.) The study was approved by the institutional ethics committee.

\section{Imaging modalities:}

1. Abdominal Ultrasound was performed using Toshiba, Ge, Siemens and Philips HD ultrasound machines with 2.5-5 MHz transducer.

2. Contrast-Enhanced CT: usingToshiba 16 slice and Somatom definition AS 128 slice from Siemens.

Statistical analysis: The collected data were organized, tabulated and statistically analyzed using statistical package for social science (SPSS), version 16 (SPSS Inc. USA), running on IBM- compatible computer.Quantitative data were represented as mean and standard deviation (SD).Qualitative data were represented as relative frequency and percentage. Comparison between groups was done by student samples ( $\mathrm{t}$ ) test for quantitative and for qualitative data using Pearson chi-square and Fisher's-exact test.Data were presented in the form of graphs, tables \& numeric presentations. P- value less than or equal to 0.05 was considered statistically significant.

\section{Results:}

Our study included 30 patients with acute pancreatitis, 16 males $(53,3 \%)$ and 14 female $(46,7 \%)$ their ages ranged between 21 years and 70 years as shown in ( figure1)

The upper abdominal pain is the most common symptom (100\%) among all patients. The patients presented with history of smoking (46.7\%), history of gall stone (46.7\%) and alcoholism (40\%) (Figure 2) biliary cause (mainly calcular gall bladder disease) was the most common etiology causing acute pancreatitis which occurred in 19 case, while 2 case had no obvious etiology for the acute pancreatitis (idiopathic etiology ),followed by dyslipidemia occurred in 7 cases then post biliary intervention cause seen in one case and posttraumatic causes was only in one patient. (Figure 3)

The laboratory investigations of the studied patients show all patients have elevated serum amylase. Most of patients have elevated serum lipase (56.7\%) and elevated serum triglycerides (36.7\%). (Figure 4) 
CT characteristics of acute pancreatitis According to the revised Atlanta classification is showed that bulky pancrease was found in about $46.6 \%$ while $53.3 \%$ had normal size pancrease, Fat stranding and obliterated fat planes was found in $93.9 \%$ while only $6.7 \%$ had clear surrounding fat planes.(Figure 5)

The type of peripancreatic collection in the current study included 17 cases with acute interstitial edematous pancreatitis 10 case with APFC and 7 cases with pseudocysts , APFC occurs before 4 weeks from the onset of symptom showing homogenouse fluid attenuation with no definable wall, while pseudocysts seen after 4 weeks showing adefinable enhanced wall (Figure 6)

Acute necrotizing pancreatitis occurred in 13 cases :7 cases with ANC and 6 cases with walled off necrosis ,ANC occurred before 4 weeks from the onset of the symptoms showing non liquefied depris (necrosis) with no definable wall while after 4 weeks this fluid typically develop well defined wall and named walled off necrosis (Figure 6)

Case 1: Male patient 50 year-old Complaint of Presented with epigastric pain radiating to the back at admission

\section{CT finding:}

Figure $(\mathrm{V})$ at the time of admission CECT scan (axial cuts) : show diffusely enlarged pancreas with peripancreatic fat stranding and edema extending along with anterior pararenal spaces of both sides as well as along mesenteric root (arrowed), interstitisl edematous pancreatitis

\section{Figure (8) Follow up after one week):}

show multiple heterogeneous lobulated fluid collections, the largest seen at pancreatic tail with pigtail drainage catheter is seen introduced in the superior-posterior aspect, pancrease is bulky with indistinct outline (arrowed), necrotizing pancreatitis (acute necrotic collection)

Figure (9) up after 21 days: show pancreas is necrotic and swollen with marked peripancreatic fat stranding , localized collection seen related to pancreatic tail, the collection reach splenic hilum and greater curvature of stomach where the tip of pigtail inserted, the collection is continuous with left paracoloic localized collection (arrowed), necrotic collection another infrapancreatic collection detected reaching the right iliac fossa, MV thrombosis detected ........Necrotizing pancreatitis with SMV thrombosis 


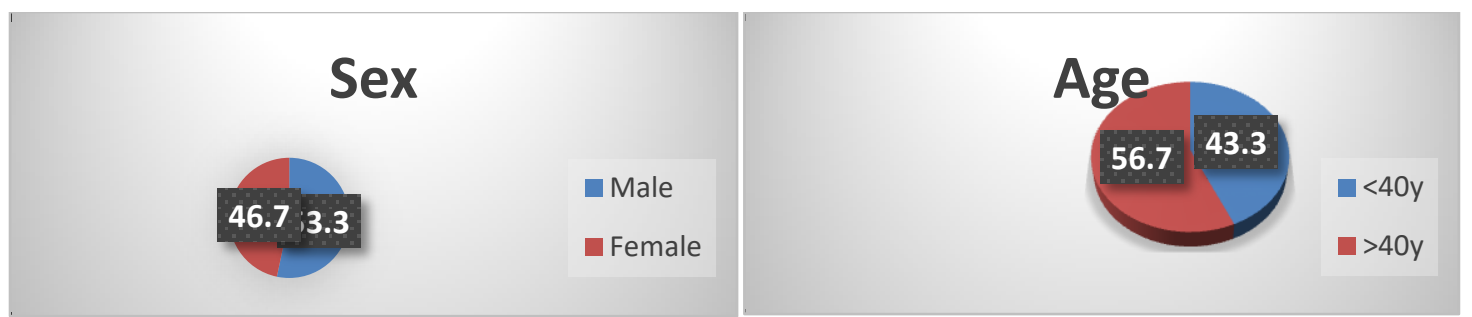

Figure 1

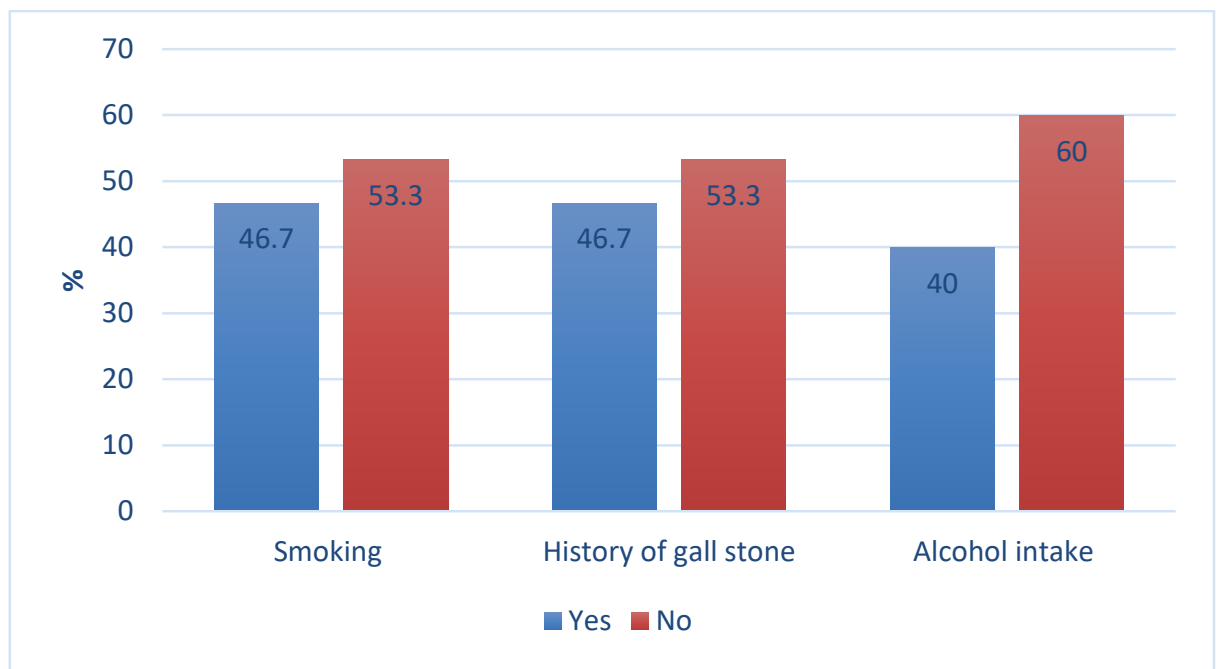

Figure 2

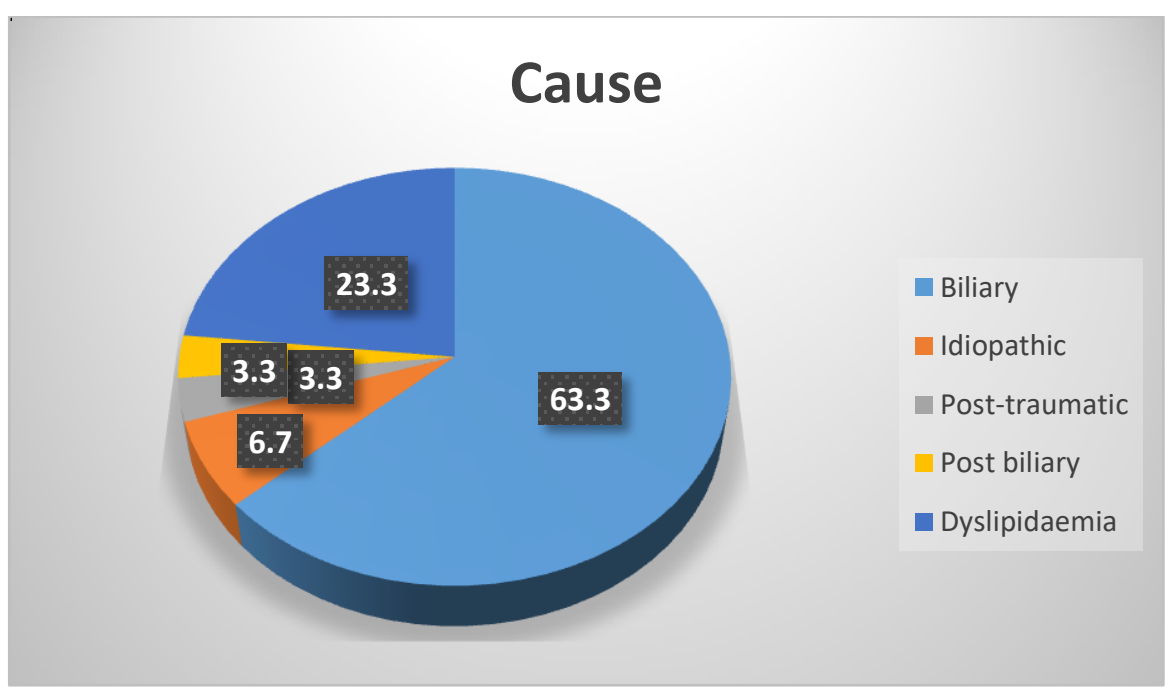

Figure 3 
Benha medical journal, vol. 39, special issue (radiology), 2022

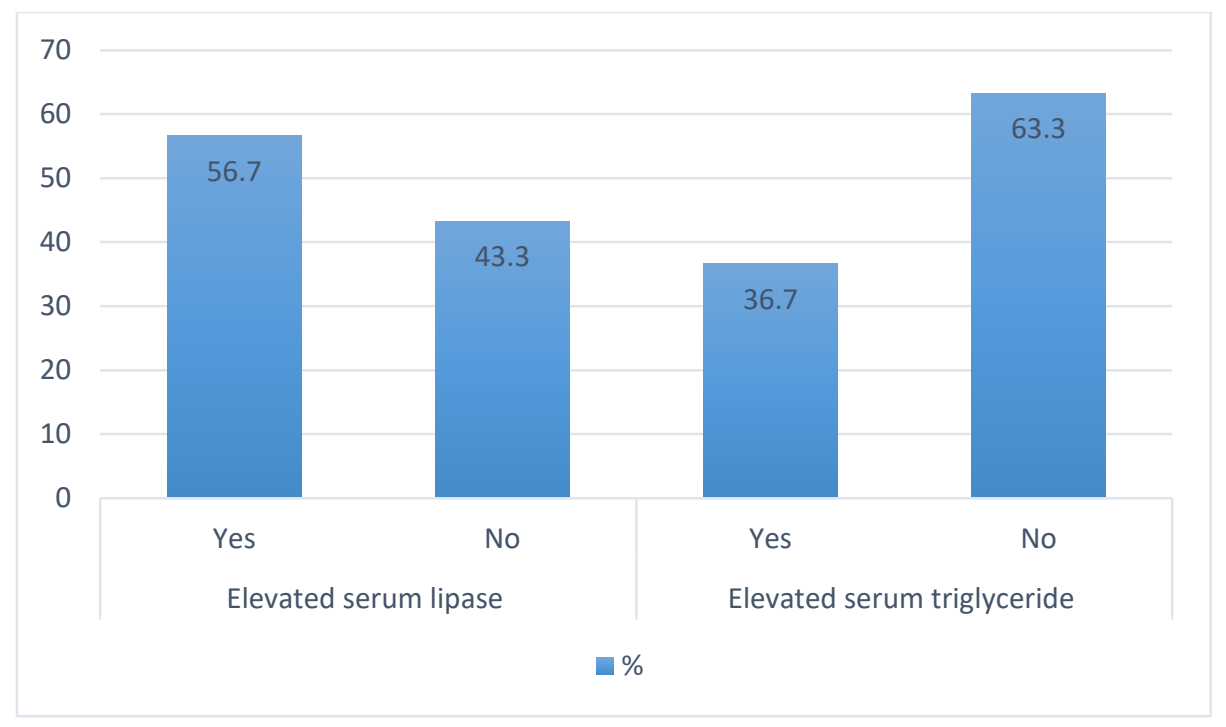

Figure 4

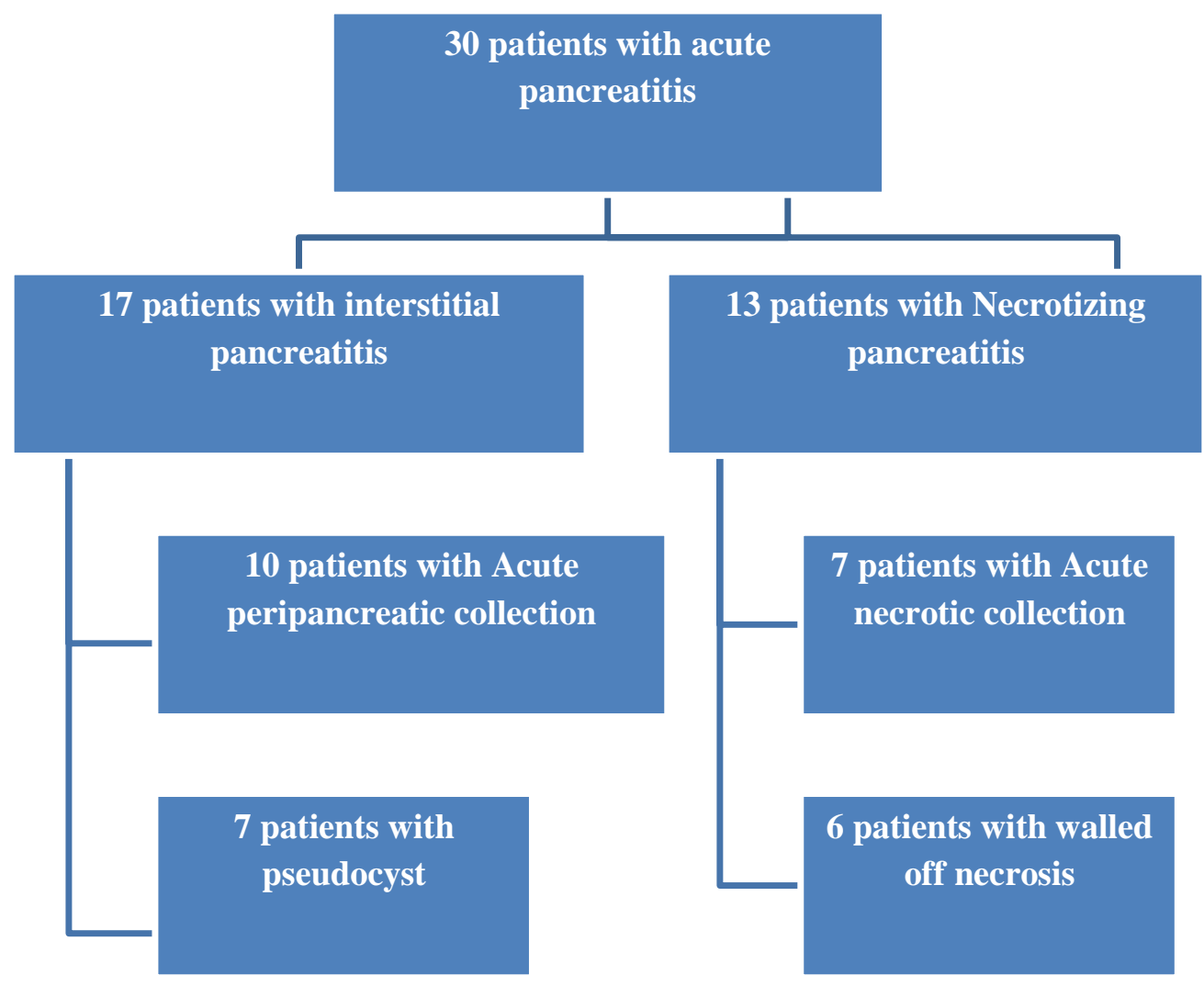

Figure 5 


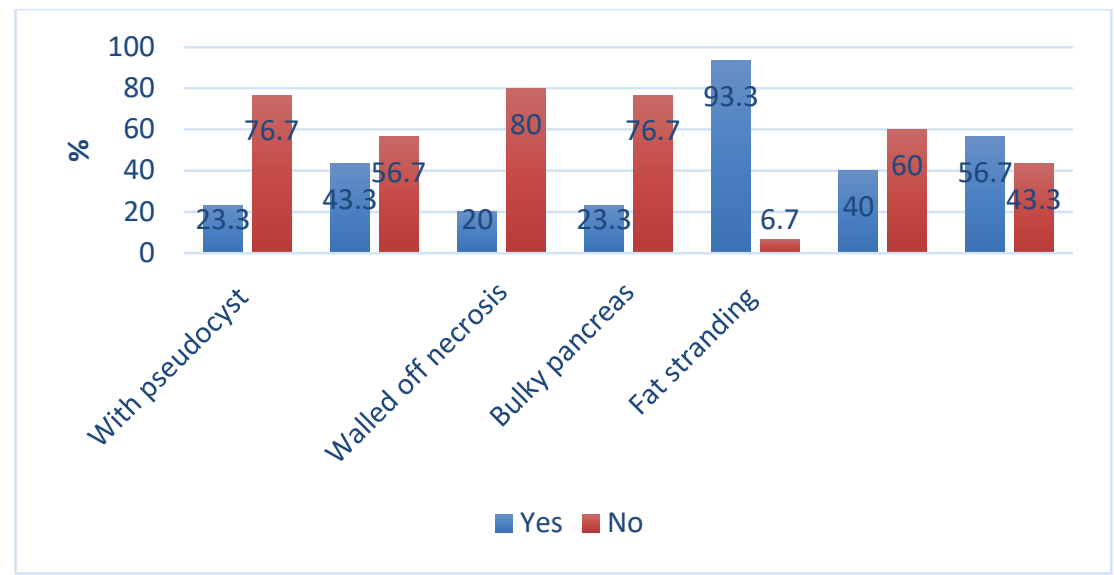

Figure 6
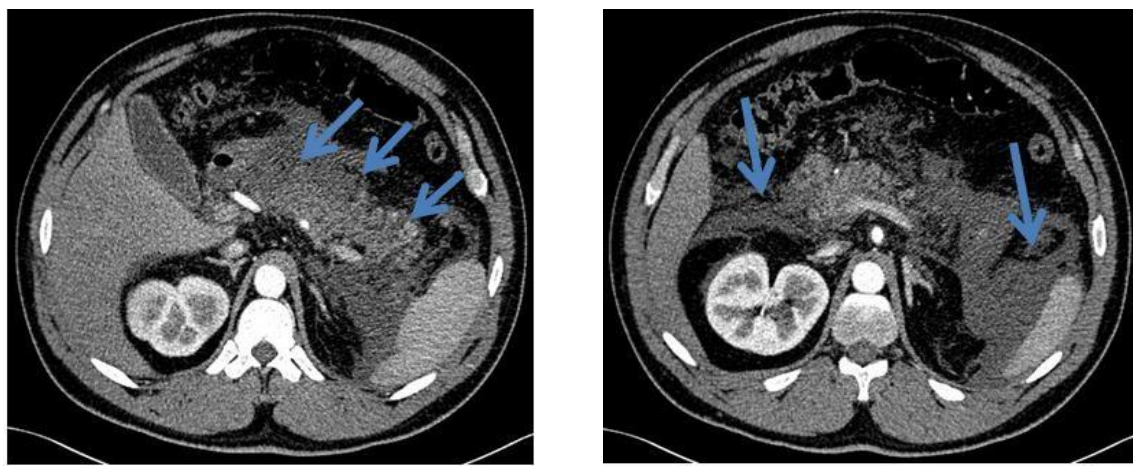

Figure 7
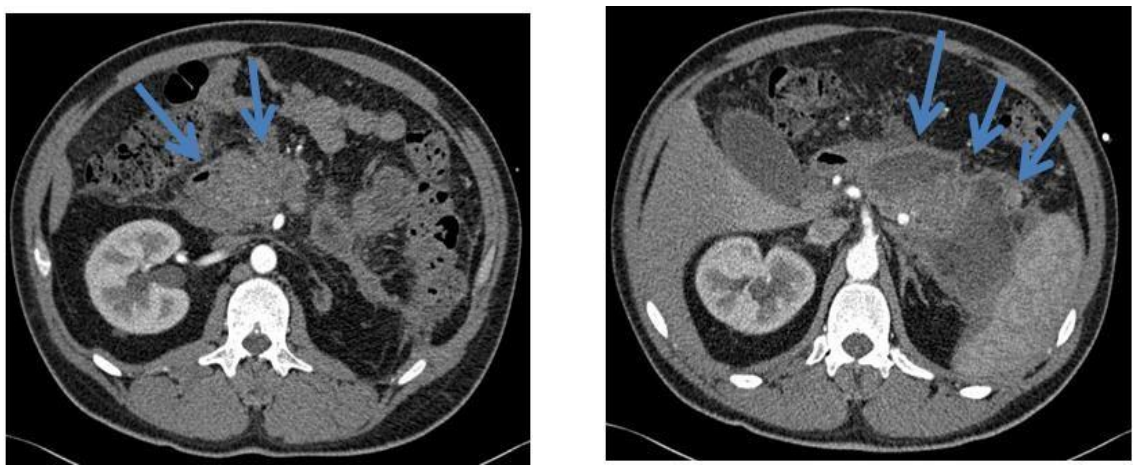

Figure 8 

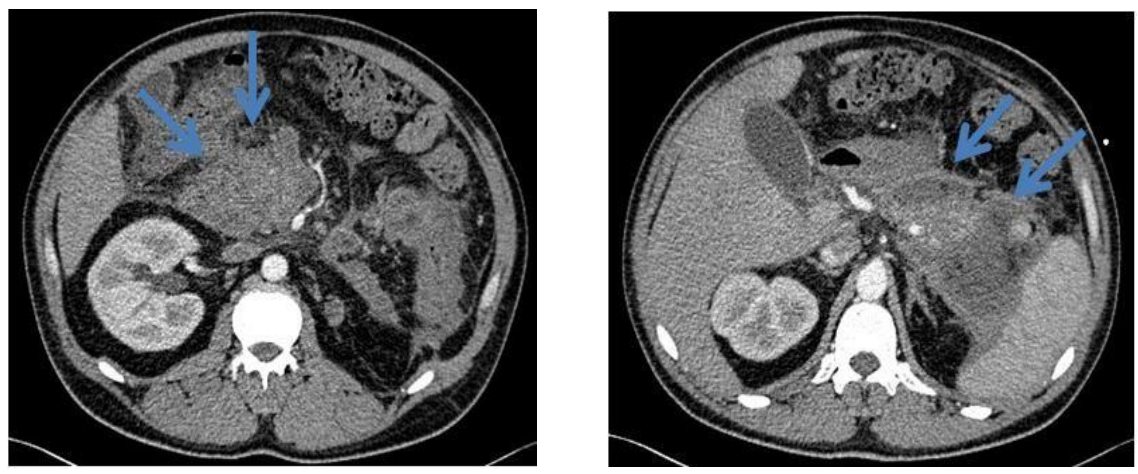

Figure 9

\section{Discussion:}

Acute pancreatitis is one of the most common diseases of the gastrointestinal tract, leading to tremendous emotional, physical, and financial human burden [8] Radiologic imaging has become increasingly important in staging and treating acute pancreatitis. [9]

This initial Atlanta classification system represented major progress, but advancing knowledge of the disease process, improved imaging, and ever-changing treatment options such as minimally invasive radiologic, endoscopic, and laparoscopic procedures soon rendered some of the definitions inadequate or ambiguous. It was found that the definitions of severity and local complications of acute pancreatitis were not used consistently and that characterization of severity based on presence of organ failure had limitations. The initial Atlanta classification system also did not include exact radiologic criteria for local complications, and controversy developed over the natural course of pancreatic and peri-pancreatic fluid collections. [10]

Revised Atlanta classification does also not consider infected pancreatic necrosis an indicator of severe disease. There is also the need to develop methods for the accurate non-invasive diagnosis of infected necrosis and evaluation of the characteristics of organ dysfunction in relation to severity and outcome. [11]

The terminology and classification scheme of acute pancreatitis proposed at the initial Atlanta Symposium was reviewed, and a new consensus statement was recently proposed. Major changes include subdividing acute fluid collections in the first 4 weeks into "acute peripancreatic fluid collection" and "acute necrotic collection" 
based on the presence of necrotic debris. Delayed fluid collections have been similarly subdivided into "pseudocyst" and "walled-off necrosis." Correct use of the new terms that describe these collections is important because they lead to different treatment decisions. [12]

The study was done on (30) patients. According to our study, Acute pancreatitis involved the following categories: interstitial edematous pancreatitis (17cases), necrotizing pancreatitis (13 cases), acute peripancreatic collection (10 cases), acute necrotic collection (7 cases), pseudocysts (7 cases), and wall of necrosis (6 cases).

In this study, The age of patients ranged from 21 to 70 years old .The study showed prevalence of acute pancreatitis in males $(53,3 \%)$ in comparison to females $(46,7 \%)$.

This result was different from the study done in 2013 [13] who stated that equal proportions of men and women develop acute pancreatitis. The risk of AP progressively increases with age. Alcoholrelated pancreatitis is more common inmen. Pancreatitis in women is more likely related to gallstones, endoscopic retrograde cholangio-pancreatography, or autoimmune diseases. Variations in age and sex distribution among geographic regions likely arise from differences in etiology.
However, this result was matching with the study done in 2017 [14] whostated that The incidence of acute pancreatitis overall was significantly higher among men (32.8) than women (27.8) and it increased across age groups.In the current study upper abdominal pain is the most common symptom (100\%) among all patients.

This matches with the study done in 1999 [15] who found that the most common symptom associated with pancreatitis is pain localized to the upper-to-middle abdomen. Patients often report that their pain radiates to the back. In this study; it was found that biliary pancreatitis was more common.

This result was matched with the study done in 2015[16] who stated that, more frequent cases of severe acute pancreatitis were observed in males with gallstones (70\%) compared to females $(\mathrm{P}<0.001)$. The aged people had a high prevalence while males were more likely to develop local and systemic complications.

This result was different from the study done in 2002 [17] who stated that, the risk of gallstones is higher in women than in men at all ages, but the difference in risk is more pronounced at younger ages. Hormonal factors are felt to be largely responsible for this association. Much of the increased risk in young women is probably related to 
pregnancy and childbearing. The prevalence of gallstones increases with increasing parity. Also, obesity is a strong risk factor for gall stone.

In the current study all patients have elevated serum amylase. Most of patients have elevated serum lipase $(56,7 \%)$ and elevated serum triglycerides $(36,7 \%)$.

This differ from the study done in 2013[18] who found that in acute pancreatitis, the plasma lipase levels increase within $4-8$ hour to double the upper limit of normal range and may remain elevated for 10 - 14 days. Because the pancreas is the only source of lipase, plasma lipase estimations are specific for pancreatic injury and may be useful in instances where the diagnosis is delayed (i.e. more than 24 hour from the onset of pain) or to differentiate acute pancreatitis from other causes of elevated serum amylase.

In this study, CT characteristics of acute pancreatitis showed bulky pancreas was found in about $46,6 \%$ while $53,3 \%$ had normal size pancreas, Fat stranding and obliterated fat planes was found in $93.9 \%$, while only $6,7 \%$ had clear surrounding fat planes.

Our study matched with the study done in 2011 [19] study who found that the patients with IEP has localized or diffuse enlargement of the pancreas, with normal homogeneous enhancement or slightly heterogeneous enhancement of the pancreatic parenchyma related to edema. On a contrast-enhanced CT study obtained within the first several days of acute onset of pancreatitis, the pancreas occasionally demonstrates increased heterogeneous enhancement of the parenchyma that cannot be characterized definitively as either IEP or ill-defined necrosis. With these findings, the presence or absence of pancreatic necrosis needs to be described initially as indeterminate

In this study we found that the peripancreatic fluid collections in the cases of interstitial edematous pancreatitis which have no necrotic changes has been classified into APFC and pseudocysts according to presence or absence of definite wall, before 4 weeks $(\leq 4)$ have no definable wall, this was found in 10 patients and termed as APFC, while "pseudocyst" which have a definable wall after 4 weeks (> 4) was formed in 7 patients.

This is matching with the study done in 2016 [20] who stated that on CECT, APFCs do not have a well-defined wall, are homogeneous in appearance, without any solid component, and are restricted by normal anatomical planes of 
retroperitoneum. If the fluid collection persists for more than four weeks, it is likely to develop into a pancreatic pseudocyst.

\section{Conclusion:}

The Revised Atlanta classification is designed to precisely describe patients with acute pancreatitis, standardize terminology across specialties, and help in treatment planning. The most important change in the Atlanta classification is the categorization of the various pancreatic collections. Treatment planning is based on severity of pancreatitis and presence or absence of infection combined with clinical signs. The revised Atlanta classification system with CT helps guide management and monitors the success of treatment.

\section{References:}

1- Yadav D, Lowenfels AB.(2013) The epidemiology of pancreatitis and pancreatic cancer. Gastroenterology 144(6):1252-1261

2- Banks PA, Bollen TL, Dervenis C.(2012)Classification Working Group. Classification of acute Pancreatitis 60293

3- Gooszen HG, Johnson CD, Sarr MG, Tsiotos GG, Vege SS.(2013) revision of the Atlanta classification and definitions by international consensus. Gut.62:102-111

4-Banks PA, Bollen TL, Dervenis C (2013) Classification of acute pancreatitis-2012: revision of the Atlanta classification and definitions by international consensus. Gut 62:102-111

5-Bollen TL.(2016) Acute pancreatitis: international classification and nomenclature. Clin Radiol 71(2): 121-133.
6- Bollen TL.(2012) Imaging of acute pancreatitis: update of the revised Atlanta classification.Radiol clin North Am 50(3): 429-445.

7-Thoeni RF (2015) Imaging of acute pancreatitis. Radiol Clin North Am 53:1189-1208

8 Demir M, Foerster U, Hoffmann V, Pelc A, Schreiter I, Chang DH, et al.(2016) Effects of early contrast-enhanced computed tomography on clinical course and complications in patients with acute pancreatitis. Zeitschrift für Gastroenterologie. ;54(07):642-6.

9- Banks PA, Freeman ML,(2006) Practice Parameters Committee of the American College of Gastroenterology. Practice guidelines in acute pancreatitis. American journal of gastroenterology. 101(10):2379-400.

10- Vege SS, Chari ST.(2005) Organ failure as an indicator of severity of acute pancreatitis: time to revisit the Atlanta classification. Gastroenterology ; 128:1133-35.

11- Windsor JA, Johnson CD, Petrov MS, Layer P ..(2015)Classifying the severity of acute pancreatitis. Pancreatology ; 15:101-4.

12- TürkvatanA, ErdenA, SeçilM and Türkoğlu MA.(2014)Fluid collections associated with acute pancreatitis: a pictorial essay. Can Assoc Radiol J. 2014; 65:260-66.

13- Yadav D and Lowenfels AB.(2013) The epidemiology of pancreatitis and pancreatic cancer. Gastroenterology ; 144(6): 1252-61.

14- Roberts SE, Morrison-Rees S, John A, Williams JG, Brown TH ..(2017)The incidence and aetiology of acute pancreatitis across Europe. Pancreatology.;17(2):155-65.

15- Ammann RW and Muellhaupt B,(1999) Zurich Pancreatitis Study Group. The natural history of pain in alcoholic chronic pancreatitis. Gastroenterology.;116(5):1132-40.

16- Malik AM.(2015) Acute pancreatitis. A more common and severe complication of gallstones in males. International journal of health sciences ; $9(2)$ : $141-5$.

17- Ko CW and Lee SP.(2002) Epidemiology and natural history of common bile duct stones and prediction of disease. Gastrointest Endosc ; 56(6): 165-9.

18- BanksPA, BollenTL, Dervenis C, Gooszen HG, Johnson CD.(2013). Classification of acute pancreatitis 2012: revision of the Atlanta 
Benha medical journal, vol. 39, special issue (radiology), 2022

classification and definitions by international consensus. Gut.;62(1):102-11.

19- Bharwani N, Patel S, Prabhudesai S, Fotheringham $\mathrm{T}$ and Power N.(2011) Acute pancreatitis: the role of imaging in diagnosis and management. Clinical radiology. ;66(2):164-75.
20- Sureka B, Bihari C, Arora A, Agrawal N, Bharathy KG, Dev K, et al.(2016) Imaging paradigm of cystic lesions in pancreas. JOP. J Pancreas (Online). 8;17(5):452-65.

To cite this article: Hesham M. Farouk, Eman F. Abdelkhalik, Nesma S. Ellithy. The Role of Multidetector CT in Revised Atlanta Classification of Acute Pancreatitis. BMFJ 2022;39 (radiology): 149-160. DOI: 10.21608/bmfj.2021.91987.1467 
